\title{
Soundness Efficient Determination Algorithm of Services Composition in Surrounding Restraint Situation Based on OPN
}

\author{
Yunfei Lu \\ Huainan Union University \\ Huainan, China \\ e-mail: 13956446726@163.com
}

\author{
Xin Gao \\ ZhongRui Communication Planning \& Designing Co.,Ltd \\ Wireless Network Design Institute \\ Guangzhou, China \\ e-mail: gxnew@qq.com
}

\begin{abstract}
It is important to analyze the soundness of the Web services composition. And the paper put forward a new method to determine the soundness. After modeling Web services area and Web surrounding area by the open Petri net, an efficient determination algorithm was offered to determine the soundness. At last, not only was the algorithm programmed, but also the paper verified the validity of the algorithm through the concrete example.
\end{abstract}

Keywords-Services Composition; Surrounding Restraint; Composability; Soundness ;OPN

\section{INTRODUCTION}

The traditional Web services composition usually does not think of the restraint between services components and the surrounding. However, the restraint and the surrounding are important in the soundness of Web services composition.

Take the video streaming media Web services for instance.

It is composed by a lot of Web services components, and if it wants to operate correctly, it must rely on the high bandwidth surrounding. So it is more and more important and necessary to analyze the soundness of Web services composition under surrounding restraint. But the Web surrounding has its own special features, and the traditional methods about modeling and analyzing can never meet the requirements. We should propose new modeling tools and analysis methods.

In the literatures [1], although the perspective that the surrounding influences the correctness of services is proposed, it did not put forward a rigorous formalized model. The literature [2,3] offered a formalized model by the services tree theory, and it also offered a determination method of the soundness of the Web services composition. But it ignored the surrounding constrain factor.

Open Petri net is a formalized mathematical tool, and if we use it to model Web services and Web surrounding, we can analyze the dynamic behavior of the model through working the open Petri net. In addition, the structure analysis and behavioral analysis functions can be combined by the open Petri net. Therefore, open Petri nets have advantages on solving the problem.

In addition, the larger the model scale is, the greater the complexity and the size of the nets are, and they increase as exponential. It appears the nets "explosion" problem. Because the "explosion" problem will lead to the difficulty of determining soundness by looking for reachable sequences in the running nets, in order to avoid the problem, the paper presents an efficient determination algorithm about soundness to reduce the time and space complexity.

\section{PRELIMINARIES}

Now, we do not have a unified and strict definition about Web services composition in academic and business. Generally, we consider Web services composition as a process that some single by Internet and other network form. Web surrounding is usually composed by user surrounding, computing surrounding and physical surrounding. We call the influence on Web services composition by Web surrounding is the surrounding restraint.

Mathematics and physics definition $1[4,5]$ : Petri Net

Petri net is a very important modeling tool for concurrent system modeling and dynamic behavior analysis which was first proposed by German C.A.Petri doctor in 1962. Petri net can not only describe the system structure, but also analyze the system dynamic properties.

A Petri Net is a $3-$ tuple $N=(S, T ; F)$, where

(1) $S \cup T \neq \phi$

(2) $S \cap T=\phi$

(3) $F \subseteq((S \times T) \cup(T \times S))$

(4) $\operatorname{dom}(F) \cup \operatorname{cod}(F)=S \cup T$

$$
\operatorname{dom}(F)=\{x \in S \cup T \mid \exists y \in S \cup T:(x, y) \in F\}
$$$$
\operatorname{cod}(F)=\{x \in S \cup T \mid \exists y \in S \cup T:(y, x) \in F\}
$$

Mathematics and physics definition 2[6,7]:: OPN

Web services composition is an open system. Web services composition and Web services components will communicate, Web services composition and the surrounding will also communicate. The open connector is the physical and logical realization of the component communication.

An Open Petri Net is a 7 -tuple $I P N=(S, T ; F)$ [8], where:
(1) ( $P \cup I \cup O, T, F)$ is a Petri net;
(2) $P$ are internal places;
(3) $T$ are transitions;
(4) $F$ are flow relations;
(5) $I$ are input places, and ${ }^{\bullet} I=\phi$; 
(6) $O$ are output places, and ${ }^{\circ} O=\phi$;

(7) $i$ are initial markings;

(8) $f$ are final markings, and $f$ are deadlock.

We call the set $I \cup O$ is the open connector places of the OPN. Two OPNs, if $\mathrm{P}_{\mathrm{N}}, \mathrm{P}_{\mathrm{M}}, \mathrm{I}_{\mathrm{N}}, \mathrm{I}_{\mathrm{M}} \mathrm{O}_{\mathrm{M}} \mathrm{O}_{\mathrm{M}}, \mathrm{T}_{\mathrm{N}}, \mathrm{T}_{\mathrm{M}}$ are pairwise disjoint $\mathrm{M}$ and $\mathrm{N}$ are disjoint.

Mathematics and physics definition 3[9,10]: A \& B

Let $\mathrm{A}$ and $\mathrm{B}$ be two OPNs, their composition is an OPN $\mathrm{A} \& \mathrm{~B}=(\mathrm{P}, \mathrm{I}, 0, \mathrm{~T}, \mathrm{~F}, \mathrm{i}, \mathrm{f})$ defined by:

(1). $P=P_{A} \cup P_{B} \cup\left(I_{A} \cap O_{B}\right) \cup\left(I_{B} \cap O_{A}\right)$

(2). $I=\left(I_{A} \backslash O_{B}\right) \cup\left(I_{B} \backslash O_{A}\right)$

(3). $O=\left(O_{A} \backslash I_{B}\right) \cup\left(O_{B} \backslash I_{A}\right)$

(4). $T=T_{A} \cup T_{B}$

(5). $F=F_{A} \cup F_{B}$

(6). $i=i_{A} \cup i_{B}$

(7). $f=f_{A} \cup f_{B}$

Two OPNs A and B are composable if and only if $\left(P_{A} \cup P_{A} \cup O_{A} \cup T_{A}\right) \cap\left(P_{B} \cup P_{B} \cup O_{B} \cup T_{B}\right)=$ $\left(I_{A} \cap O_{B}\right) \cup\left(O_{A} \cap I_{B}\right)$.

Mathematics and physics definition 4[11]: Soundness of the INP

Let $\mathrm{N}$ be an open Petri net. The skeleton of $\mathrm{N}$ is defined as the Petri net $S(N)=\left(P_{N}, T_{N}, F\right)$ with $F=F_{N} \cap\left(\left(P_{N} \times T_{N}\right) \cup\left(T_{N} \times P_{N}\right)\right)$.We use $R(N)$ as a shorthand notation for $R\left(S(N), i_{N}\right)$. N is called sound if for any marking $\mathrm{m} \in R(N)$ we have $S(N): \mathrm{m} \stackrel{*}{\longrightarrow} f_{N}$.

In many cases, multiple Web services areas share a Web surrounding area. As shown in Figure 1, we have three components A, B, and C. The composition of A with B is sound, as well as the composition of B with C. However, the composition of the three is not sound.

Mathematics and physics definition 5[12,13]: \& $\&_{A, B}$

A and $\mathrm{B}$ are two composable OPNs, $\&_{A, B}$ holds if and only if :

$$
\begin{aligned}
& \forall m \in R(A \& B), \sigma \in\left(T_{A}\right) * \\
& :\left(A: m \mid P_{A}-\underline{\sigma} \rightarrow f\right) \\
& \Rightarrow\left(\exists \sigma \in\left(T_{A} \cup T_{B}\right) *:\right. \\
& \left(A \oplus B: m-\longrightarrow \frac{\sigma}{\sim} \rightarrow f_{A}+f_{B}\right) \wedge \tilde{\sigma} \mid T_{A}=\sigma
\end{aligned}
$$

$\mathrm{A}$ and $\mathrm{B}$ are two composable OPNs, if A satisfies soundness and $\&_{A, B}$ holds, A \& B satisfies soundness. Let A, B, C are three pairwise composable OPNs such that $A$ and $C$ have disjiont open connector places, A, B, C pairwise composable, if A \& B satisfies soundness, and $\&_{B, C}$ holds, A \& B \& $C$ also satisfies soundness.

Mathematics and physics definition 6: Web services area
A Web services area (WA) is a 4tuple $\mathbb{H A}=\left(S_{I}, S_{o}, S_{C}, C_{I}\right)$, where $S_{I}$ is a services input, $S o$ is a services output, $S C$ is a services component and $C_{I}$ is a communication connector.

Mathematics and physics definition 7: Web surrounding area

A Web surrounding area (SA) is a 4tuple $S \mathrm{~A}=\left(E_{I}, E_{o}, E_{C}, C_{I}\right)$, where $E_{I}$ is an surrounding input, $E O$ is a surrounding output, $E C$ is a surrounding component and $C_{I}$ is a communication connector.

Definition 8: Two Models Mapping

Let $W \mathrm{~A}=\left(S_{I}, S_{o}, S_{C}, C_{I}\right)$ be a Web services area, $S \mathrm{~A}=\left(E_{I}, E_{o}, E_{C}, C_{I}\right)$ be a Web surrounding area and $M=(P, I, O, T, F, i, f)$ be an open Petri Net. If $S_{I} \subseteq \mathrm{i}, S_{o} \subseteq f, S_{C} \subseteq \mathrm{P}$ and $C_{I} \subseteq I \cup O$, sA should be mapped to an OPN and if $E_{I} \subseteq \mathrm{i}, E_{o} \subseteq f, E_{C} \subseteq \mathrm{P}$ and $E_{I} \subseteq I \cup O$,EA should be mapped to an OPN.

Through open connector places, Web surrounding communicates with Web services, thus an interrelated whole is formed structurally and behaviorally. Because of the communication, Web surrounding inevitably constrains Web services behavior which is reflected by the correctness of composition.

\section{SOUNDNESS EFFICIENT DETERMINATION ALgORITHM OF SERVICES COMPOSITION IN SURROUNDING RESTRAINT SITUATION BASED ON OPN}

Web services area, Web surrounding area and open Petri nets all have input terminal, output terminal, open connector terminal and the internal components which have the logical connection among them. That is they are similar on the structure and function. Hence, the components of Web services area and Web surrounding area can be mapped to the corresponding components of open Petri nets.

The premise of the Web surrounding restraint is that Web surrounding and Web services can compose, so we must determine whether Web surrounding and Web services can compose firstly. A and B are two OPNs, if (PA $\cup$ IA $\cup$ $\mathrm{OA} \cup \mathrm{TA}) \cap(\mathrm{PB} \cup \mathrm{IB} \cup \mathrm{OB} \cup \mathrm{TB})=(\mathrm{IA} \cap \mathrm{OB}) \cup$ $(\mathrm{OA} \cap \mathrm{IB})$ holds; Then $\mathrm{A}$ and $\mathrm{B}$ can compose[14,15].

In the actual application, Web services area/Web surrounding composition has large number and scale, so the open Petri net model also become very complex and massive. It is almost impossible to run the open Petri net with a large scale. Hence this paper proposes a new high efficiency determining algorithm about soundness of Web services composition under surrounding restraint.

Efficient determination algorithm: soundness decision algorithm for arbitrary Web surrounding area/Web services area composition process.

Input: $\mathrm{D} 1, \ldots \ldots . \mathrm{Dn}$ are Web surrounding areas/Web services areas 
Output: decision outcome

(1). By definition 5, put D1,.....Dn map into OPN: $A 1, \ldots . . A n$, then execute step (2).

(2). By definition 6,if $\left(P_{i} \cup P_{i} \cup O_{i} \cup T_{i}\right) \cap\left(P_{i(c)} \cup P_{i(c)} \cup O_{i(c)} \cup T_{i(c)}\right)$ $=\left(I_{i} \cap O_{i}\right) \cup\left(O_{i(c)} \cap I_{i(c)}\right)$

holds, $A 1, \ldots . . A n$ are composable, then execute step (3). Otherwise algorithm terminates and returns the result that they do not satisfy soundness.

(3). $A 1, \ldots . . A n$ is an area tree with the parent $\mathrm{A} 1$, if and only if let $\mathrm{c}:\{2, \ldots \ldots n\} \rightarrow\{1, \ldots . . n-1\}$ be such that: $[16,17]$ :

$$
\begin{aligned}
& \forall \mathrm{i} \in\{2, \ldots \ldots \mathrm{n}\}: \mathrm{c}(\mathrm{i})<\mathrm{i} \\
& \forall 1 \leq i<j \leq n: i=c(j) \Rightarrow I_{A \mathrm{i}} \cap O_{A \mathrm{j}} \neq \theta \vee O_{A \mathrm{i}} \cap I_{A \mathrm{j}} \neq \theta \\
& \forall 1 \leq i<j \leq n: i=c(j) \Rightarrow \\
& { }^{I}{ }_{A \mathrm{i}} \cap O_{A \mathrm{j}} \neq \theta \vee O{ }_{A \mathrm{i}} \cap I_{A j} \neq \theta \\
& \text {, then execute step (4). }
\end{aligned}
$$

(4). If $A 1, \ldots . . A n$ is an area tree with the parent $\mathrm{A} 1$, A1 is sound and $\&_{A i, A c(i)}$ holds, $A 1 \& \ldots \ldots \& A n$ is also sound, then execute step (5). Otherwise algorithm terminates and returns the result that they do not satisfy soundness.

(5). Algorithm terminates and returns the result that composition of $A 1, \ldots . . A n$ satisfies soundness.

\section{EXAMPLE ANALYSIS AND AUTOMATIC ANALYSIS SYSTEM DEVELOP}

We usually use the operation times of transition $t$ and the marking state of the place to estimate the time complexity and the space complexity respectively in the OPN model. Every time the transition t runs is recorded as a unit running time, and the number of the place in a reachable sequence is recorded as a space consumption unit.

We do an experimental simulation which compares the algorithm in the paper and the traditional algorithm. The specific process is as follows:

The services generated randomly are used as test cases which follow OWL-S and other international standards.

Test surrounding and devices:

CPU: AMD Phenom FX $2.4 \mathrm{GHz}$ (4 core);

RAM:8GB*2;

Operation System : Windows Server 2003(64bit).

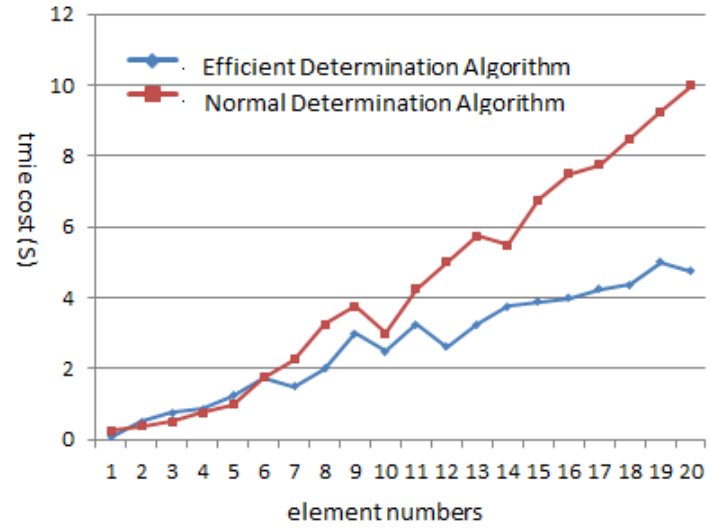

Figure 1. Time cost contrast experiment

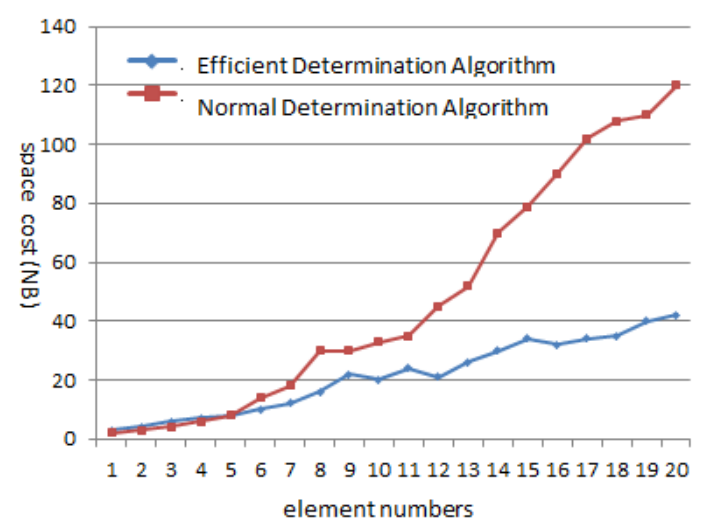

Figure 2. Space cost contrast experiment

As can be seem from the results, with the increase in the number of the services, the time and the space complexity of the algorithm in the paper and in the traditional algorithm grow linearly and exponentially respectively. It shows that the algorithm in the paper is more effective to reduce the time and space complexity and solve the nets "explosion" problem.

To realize the automatic analysis, the paper uses JAVA to program the algorithm by eclipse platform. Because the map from Web surrounding area/Web services area into the OPN involves semantic, logic analysis and other factors, we use artificial way to edit the Web surrounding area/Web services area model according to the XML standard, in order to realize the map from the physical model to the OPN model. After software imports an XML file, Visual OPN models can automatically be generated. Then we determine the soundness of the OPN according to the above algorithm, finally the analysis operation results and conclusions are given. 


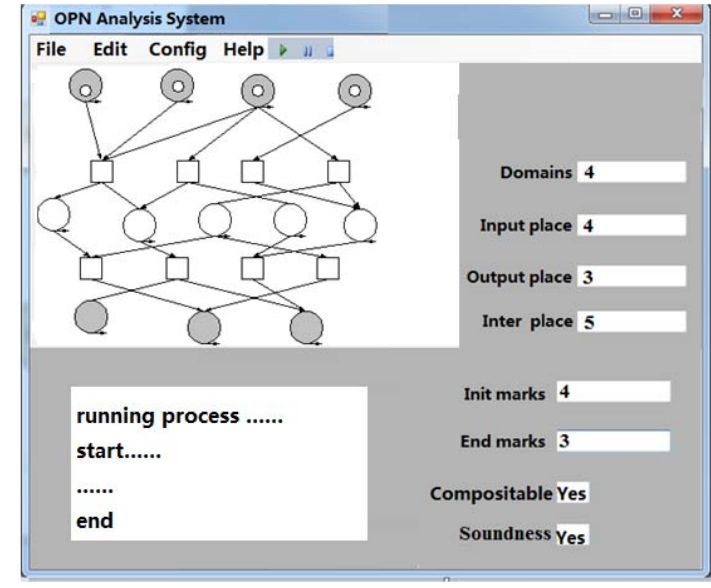

Figure 3. OPN automatic analysis system

From use OPN analysis system we can efficient and accurate analysis the soundness of services composition in surrounding restraint situation. When services composition has huge element number, OPN analysis system has more obvious advantage.

\section{CONCLUSION}

In order to consider the surrounding restraint and solve "explosion" problem in time and space complexity, the paper uses open Petri net to model and analyze Web services composition under the surrounding restraint, and also presents a determining algorithm about soundness of Web services composition. Finally, the experimental simulation result shows that the method in the paper is better and more efficient than the method of finding reachable sequences in running nets. Transplanting the determination algorithm to commercial Web protocols and languages is our future research emphasis.

\section{ACKNOWLEDGMENT}

We would like to thank the support of the Provincial demonstrative practicing and training center, Practicing and training center of Electrical engineering and stage achievement of demonstrative practicing and training center under the provincial quality project.

\section{REFERENCES}

[1] Van de Wouw, N., Nesic, D., Heemels, W. P. M. H.A discrete-time framework for stability analysis of nonlinear networked control systems. Automatica. 48, 1144-1151,2012.

[2] N.Lohmann, O.Ratsimor, V.Korolev, A.Joshi.Finin, Ahlem Ben Hassine and K.M.van Hee. From Public Views to Private Views: Correctness-by-Design for Services. Munich: springer, 2007:139-153.

[3] Kees M.van Hee, Ahlem Ben Hassine, A.Joshi.Finin, K.M.van Hee and W.M.P.vander Aalst. Compositional Services Trees. Munich: springer, 2011:287-289.

[4] Guo, C. X., Huang, C.J., Qian, Y., Liu, J. H., Jiang, X. C.: The Electroquasistatic Field Simulation and Analysis for the Insulation Defects in XLPE Power Cables. International Review of Electrical Engineering-IREE. 4, 1413-1417,2009.
[5] Dam, H. K., Winikoff, M.: An agent-oriented approach to change propagation in software maintenance. Autonomous Agents and MultiAgent Systems. 23, 384-452,2011.

[6] Postoyan, R., Nesic, D.: On emulated nonlinear reduced-order observers for networked control systems. Automatica. 48, 645652,2012 .

[7] Lopez-Echeverria, D., Magana, M. E.: Variable Sampling Approach to Mitigate Instability in Networked Control Systems with Delays. IEEE Transactions on Neural Neural Networks and Learning Systems. 23, 119-126, 2012.

[8] P. Massuthe, W.M.P.vander Aalst, K.M.van Hee and M. Weske. Compositional services trees. Eindhoven: Technische Universiteit Eindhoven, 2009:23-25.

[9] Emeka, E., Jia, B., Riley, D., Weng, J. N., Wei, Y., Xue, Y., Koutsoukos, X., Sztipanovits, J.: NCSWT: An integrated modeling and simulation tool for networked control systems. Simulation Modeling Practice and Theory. 27, 90-111,2012.

[10] Zeng, W., Chow, M.Y.: Optimal Tradeoff Between Performance and Security in Networked Control Systems Based on Coevolutionary Algorithms. IEEE Transactions on Industrial Electronics. 59, 30163025, 2012.

[11] Fei, G.Y., Gao, J., Owodunni, O., Tang, X. Q.: A method for engineering design change analysis using system modeling and knowledge management techniques. Computer Integrated. 24, 535551,2011 .

[12] Eramo, R., Malavolta, I., Muccini, H., Pierantonio, A.: A modeldriven approach to automate the propagation of changes among Architecture Description Languages. Software and Systems Modeling. $11,29-53,2012$

[13] Vanhatalo, J., Völzer, H., Koehler, J.: The refined process structure tree. Data \& Knowledge Engineering. 68, 793-818,2009.

[14] N. Lohmann, P.Massuthe, C. Stahl, and D.Weinberg. Analyzing Interacting BPEL Processes. New York: Computer Science, 2011:1732.

[15] Weber, B., Reichert, M., Rinderle-Ma, S. : Change patterns and change support features - enhancing flexibility in process-aware information systems. Data \& Knowledge Engineering. 66, 438466,2008 .

[16] Ahlem Ben Hassine, A.Joshi.Finin, K.M.van Hee and W.M.P.vander Aalst. A Restraint-Based Approach to Horizontal Web Services Composition Munich: springer, 2009:130-143.

[17] M. Beisiegel, N.Lohmann, O.Ratsimor, V.Korolev, A.Joshi Finin and Ahlem Ben Hassine. An SOA-Based Architecture Framework[J]. International Journal of Business Process Integration and Management, 2011, 12:91-91.

[18] F. Casati, H. Kuno, V. Machiraju, W.M.P. vander Aalst and M.Beisiegel. Web Services Concepts, Archi-tectures and Applications. Munich: Springer, 2011:342-345.

[19] G.Decker, N.Lohmann, O.Ratsimor and M. Weske. Local enforceability in interaction petri nets. New York: Computer Science, 2009:195.

[20] Xin Gao.Restraint-aware Correctness Analyzing of Composite Web Services Based on Open Petri Net. New York: dcabes2011, 2011:195. 